\title{
PERFIL AUDIOLÓGICO DO USUÁRIO IMPLANTE COCLEAR E APARELHO DE AMPLIFICAÇÃO SONORA INDIVIDUAL NA ORELHA CONTRALATERAL: RESULTADOS PRELIMINARES
}

\author{
Audiologic profile of hearing aids users in the contralateral ear \\ of a cochlear implant: preliminary results
}

Cíntia Tizue Yamaguchi ${ }^{(1)}$, Maria Valéria Schmidt Goffi-Gomez ${ }^{(2)}$

\begin{abstract}
RESUMO
Objetivo: conhecer o perfil residual audiológico dos usuários e não usuários de Aparelho de Amplificação Sonora Individual (AASI) contralateral ao Implante Coclear (IC) no Hospital das Clínicas da Faculdade de Medicina da Universidade de São Paulo (HCFMUSP). Métodos: foram avaliados 18 pacientes adultos, pós-linguais que preencheram os critérios estipulados, respondendo à pergunta "Você usa ou já usou AASI na orelha contralateral ao IC. Foi realizada a análise de média tonal liminar dos 18 pacientes. Resultados: os pacientes do Grupo de Implante Coclear da Divisão de Otorrinolaringologia da Faculdade de Medicina da Universidade de São Paulo, que não usam AASI contralateral ao IC, têm perfil residual audiológico médio de $122.5 \mathrm{~dB}$. Os pacientes que se beneficiam do uso de AASI associado ao IC tem perfil residual audiológico médio de $101.25 \mathrm{~dB}$. Conclusão: os pacientes não usuários de AASI contralateral têm audição residual significativamente pior do ponto de vista clínico que os pacientes que usam AASI contralateral associado ao IC na Divisão de Otorrinolaringologia do HCFMUSP.
\end{abstract}

DESCRITORES: Implante Coclear; Auxiliares de Audição; Deficiência Auditiva

\section{INTRODUÇÃO}

O implante coclear (IC) é atualmente o equipamento de reabilitação auditiva padrão para perda auditiva neurosenssorial de grau severo a profundo bilateral ou profundo bilateral, realizado atualmente em vários centros especializados no mundo todo ${ }^{1}$. Em 1977, nos Estados Unidos, um estudo multicêntrico concluiu que os implantes cocleares são benéficos, e assim, foi aprovado pelo Instituto Nacional de saúde americano $(\mathrm{NIH})^{2}$. No Brasil, o precursor

(1) Fonoaudióloga; Fonoaudióloga no Grupo de Implante Coclear do Hospital das Clínicas da Faculdade de Medicina da Universidade de São Paulo, HCFMUSP, São Paulo, SP; Mestranda na Clínica de Otorrinolaringologia do Hospital das Clínicas da Faculdade de Medicina da Universidade de São Paulo.

(2) Fonoaudióloga; Chefe da Equipe de Fonoaudiologia do Grupo de Implante Coclear do Hospital das Clínicas da Faculdade de Medicina da Universidade de São Paulo, HCFMUSP, São Paulo, SP; Doutora pela Universidade Federal de São Paulo.

Conflito de interesses: inexistente da disseminação do IC foi o Prof. Dr. Pedro Mangabeira Albernaz, que iniciou seus estudos na área em 1978, quando se associou ao Ear Research Institute, de Los Angeles, participando de pesquisas como co-investigador ${ }^{3}$.

A partir daí, o Brasil iniciou suas pesquisas e a criação de centros, e em particular, o Grupo de Implante Coclear da Faculdade de Medicina da Universidade de São Paulo (FMUSP), que em 1989, a Disciplina de Otorrinolaringologia em conjunto com a Divisão de Bioengenharia do Instituto do Coração da FMUSP, visava estimular a tecnologia nacional para oferecer aos pacientes com deficiência auditiva, um IC de baixo custo. Em 1990, criaram o implante coclear monocanal FMUSP-1, e a partir desta iniciativa, formou-se o Grupo de Implante Coclear do HC-FMUSP ${ }^{4}$. Os resultados obtidos com o IC têm se mostrado, importantes para a comunicação ${ }^{5-7}$, ainda que o implante coclear forneça ao paciente a audição monoaural.

No entanto, alguns autores ${ }^{8-10}$ enfatizam que a audição unilateral é insuficiente em vários aspectos relacionados à aprendizagem, à localização sonora, 
aumento de loudness, reconhecimento de fala em situação de ruído. Baseando-se nesses dados, o uso de aparelho de amplificação sonora individual (AASI) contralateral ao IC foi recomendado pelo Consenso Internacional em $2005^{10}$. A binauralidade pode oferecer benefícios na melhora da percepção de palavras e maior acesso aos sons, e sugerem a melhora também na oralização e localização sonora em crianças ${ }^{9,11}$.

Os autores que estudaram a bimodalidade (IC mais AASI na orelha não-implatada), concluíram que o paciente usuário de IC associado ao AASI na orelha contralateral tem melhor desempenho em testes de sentenças, de números e de monossílabos, no silêncio ${ }^{12}$. Além de considerarem a bimodalidade, a quantidade de resíduo auditivo também é determinante para a boa adaptação de AASI como recurso associado ao IC ${ }^{13,14}$.

Este trabalho tem como objetivo estabelecer o perfil residual audiológico dos usuários e não usuários de AASI contralateral ao IC, de pacientes da Equipe de Implante Coclear da Divisão de Otorrinolaringologia da Faculdade de Medicina da Universidade de São Paulo.

\section{MÉTODOS}

Foram avaliados 19 pacientes adultos, com deficiência auditiva neurossensorial bilateral de grau severo a profundo, usuários de IC, que preencheram os seguintes critérios:

- Usuário de implante coclear multicanal unilateral, segundo os critérios do HCFMUSP ${ }^{15}$.

- Usuários adultos, acima de 18 anos;

- Surdez pré e/ou pós-lingual.

- Os critérios de exclusão foram:

- Algum tipo de impedimento que impeça a audição contralateral;

- Nível de linguagem insuficiente para responder ao protocolo;

- Impossibilidade de contato.

Os pacientes assinaram o termo de consentimento avalizando a participação na pesquisa. Foi realizada audiometria tonal liminar com fone na oreIha contralateral ao implante coclear. Para o cálculo da média tonal foram usados os critérios propostos por Davis \& Silverman ${ }^{16}$. Para os pacientes com ausência total de limiares tonais, foi estipulado o valor de $125 \mathrm{dBNA}$, para o cálculo da média tonal, por exceder os limites máximos do equipamento (Madsen Midimate 622), utilizado na audiometria tonal liminar na orelha contralateral ao IC.

A seleção dos pacientes foi de forma aleatória, conforme agendamento de retornos para programação, no centro de implante coclear do HCFMUSP, no período de 06/2006 a 08/2006. Um paciente foi excluído do estudo, após a avaliação, devido quadro de neurinoma no nervo acústico na orelha contralateral ao IC. Resultando um total de 18 pacientes participantes, sendo 12 do sexo masculino e seis do sexo feminino.

Todos os participantes responderam à pergunta: "Você usa AASI do outro lado do IC?" Com base na pergunta, os pacientes foram distribuídos em dois grupos, G1 pacientes usuários de AASI contralateral e G2 pacientes não usuários.

Esta pesquisa foi aprovada pelo Conselho de Ética em Pesquisa do HCFMUSP sob protocolo 779/06.

Os dados foram analisados usando ferramentas da estatística descritiva e o teste $t$ de student. O nível de significância adotado neste estudo foi $p<0,05$.

\section{RESULTADOS}

Dos $18(100 \%)$ pacientes que foram pesquisados neste estudo preliminar, 14 (77\%) não fazem uso de AASI contralateral ao IC, e quatro (22\%) dos pacientes pesquisados usam AASI contralateral ao IC.

Analisando os resultados da audiometria tonal na orelha contralateral ao IC, pela média tonal, baseada em Davis e Silverman ${ }^{16}$ em dBNA dos pacientes pesquisados pode-se chegar aos valores na Tabela 1.

Dos pacientes que usam AASI contralateral ao IC, apresentam a média de $0,5 \mathrm{a} 2 \mathrm{kHz}$ de 101,5 dBNA, tendo como mínimo 100 dBNA e como máximo $105 \mathrm{dBNA}$. Paralelamente, os pacientes que não utilizam o AASI contralateral ao IC, possuem a média tonal de 0,5 a $2 \mathrm{kHz}$ de $122,5 \mathrm{dBNA}$, tendo como mínimo $95 \mathrm{dBNA}$ e como máximo $125 \mathrm{~dB}$. Ou seja, os limiares tonais de usuários de $A A S I+I C$ variam de $100 \mathrm{~dB}$ a $105 \mathrm{~dB}$, e de nãousuários, de $95 \mathrm{~dB}$ à $125 \mathrm{~dB}$.

A análise das médias e da mediana comparadas entre os grupos foram realizadas através do teste $t$ de student $\mathrm{p}<0,0001$ e Mann-Whitney com $p=0,0029$, respectivamente.

$\mathrm{Na}$ Tabela 2 pode-se analisar a influência do novo critério de seleção dos pacientes do HCFMUSP ${ }^{15}$ o qual permite que indivíduos com perda de grau severo a profundo sejam candidatos ao IC - antes apenas pessoas com perda de grau profundo bilateral eram candidatos ao IC - mostra maior número de indivíduos que usam o IC associado ao AASI (23\%). 
Tabela 1 - Limiar médio (mediana, mínimo e máximo) em dBNA dos pacientes do grupo 1 (usuários de AASI contralateral) e do grupo 2 (não usuários)

\begin{tabular}{lcccc}
\hline & G1 & G2 & t de student & Mann-Whitney \\
\hline Média & 101,25 & 122,57 & & \\
Desvio Padrão & 2,50 & 7,76 & & \\
Mínimo & 100 & 95 & $\mathrm{p}<0,0001$ & $\mathrm{p}=0,0029$ \\
Máximo & 105 & 125 & & \\
Mediana & 100 & 125 & & \\
\hline
\end{tabular}

O teste $t$ de student foi significante com $p$ valor $<0,0001$

Foi realizado também o teste de Mann-Whitney com $p=0,0029$

Tabela 2 - Comparação de uso de IC associado ao AASI entre pacientes implantados até 2003 e depois de 2003

\begin{tabular}{cccc}
\hline Ano & Usuários de AASI e IC & Não usuários & Total \\
\hline $1999-2003$ & 1 & 4 & 5 \\
A partir de 2004 & 3 & 10 & 13 \\
\hline
\end{tabular}

\section{DISCUSSÃO}

O Consenso Internacional de Implante Coclear bilateral e Estimulação Bimodal ${ }^{10}$ reafirma a que a adaptação de AASI contralateral ao IC, deve ser feita em pacientes com resíduo auditivo e bom desempenho com o AASI usado contralateralmente ao IC.

A possibilidade da binauralidade é um fator relevante para o uso do IC associado ao AASI. Autor ${ }^{8}$ aborda a audição monoaural como insuficiente, num estudo baseado em crianças com perdas auditivas unilaterais e seus desempenhos escolares. E conclui que a binauralidade é muito importante tanto para a comunicação quanto para o aprendizado. De fato, nessa casuística, alguns pacientes, informalmente, relatam que o uso do AASI associado ao IC proporciona benefícios no dia-a-dia, pois o som fica "mais aberto". Para outro autor ${ }^{21}$ a assimetria de entrada auditiva entre as orelhas pode interromper ou prejudicar o processamento de som binaural.

Um estudo ${ }^{12}$ comparou os sujeitos da pesquisa com IC isolado e IC associado ao AASI. Nos testes de sentenças, a média de percepção de fala em pacientes com IC isolado foi de $79 \%$, enquanto a média de percepção de fala de pacientes com IC associado ao AASI foi de $88,1 \%$. Os testes de monossílabos para IC isolado mostraram médias de $37,2 \%$ e $48,7 \%$ para pacientes com IC associado ao AASI. Resultados que reforçam a importância do AASI utilizado na orelha contralateral ao IC.
Neste estudo, verificou-se que dos 18 pacientes com implante coclear, apenas quatro usam AASI combinado ao IC. O questionamento principal é por qual, ou quais motivos a maioria dos pacientes não se beneficia do uso contralateral do AASI, em relação ao IC. Várias possibilidades podem ser levantadas, para justificar o não uso de AASI contralateral ao IC, como ausência de resíduo auditivo ou pouco resíduo auditivo, falta de orientação no pré-operatório, por não possuírem $A A S I$, ou AASI quebrado, AASI ultrapassado, assim como as regulagens dos mesmos. Neste trabalho foi enfatizado o perfil audiológico residual, pois servirá de base para estudos futuros, sobre a não utilização de IC associado ao AASI. A média tonal do grupo usuário de IC associado ao AASI foi de $101,5 \mathrm{~dB}$, e dos pacientes não usuários a media foi de $122,5 \mathrm{~dB}$. De fato, o resíduo auditivo contralateral ao IC parece requisito importante, para a bimodalidade, pois sem benefício com AASI o paciente tende a desmotivação.

Em estudo realizado em 2004 no Brasil ${ }^{18}$, foi pesquisado sobre o uso combinado de IC associado ao AASI na orelha oposta. Seis adultos com média tonal liminar de 0,5 a $2 \mathrm{kHz}$ de $110 \mathrm{~dB}$ não mostraram melhora significativa em relação a limiares auditivos, índices de reconhecimento de monossílabos, sílabas sem sentido entre usuário de IC isolado e IC mais AASI. Os autores reforçam ainda, a importância do resíduo auditivo, como justificativa para os resultados obtidos. Os pacientes do HCFMUSP que não são usuários de AASI contralateral ao IC apresentaram média tonal liminar superior aos pacientes desse estudo. 
Em pesquisa realizada com 16 crianças observou-se que o uso de IC associado ao AASI contralateral ${ }^{11}$ contribuiu significativamente para percepção de fala, localização sonora e na comunicação do dia-a-dia das crianças, desde que o AASI esteja devidamente ajustado. A média de 0.5 a $2 \mathrm{kHz}$ dessas crianças foi de 104 dBNA. Estudos realizados também com crianças revelaram que o uso de AASI na orelha não implantada deveria ser padrão para população pediátrica usuária de IC unilateral como forma de promover a audição binaural e seus benefícios ${ }^{22,23}$.

Em um trabalho realizado ${ }^{19} \mathrm{com} 18$ crianças, os autores objetivaram determinar o efeito da experiência auditiva binaural (IC + AASI) para percepção de fala, localização horizontal e performance funcional do dia-a-dia com IC isolado e IC associado ao AASI contralateral. Dois grupos foram formados, o primeiro de oito pacientes experientes no uso de IC associado ao AASI, com média tonal liminar $(500 \mathrm{~Hz}, 1 \mathrm{kHz}$ e $2 \mathrm{kHZ}$ ) de $101.1 \mathrm{dBNA}$, e o segundo grupo formado por 10 crianças novas usuárias de IC mais AASI. Estas que não usavam AASI e foram adaptadas tinham média tonal liminar de 105.7 dBNA. Os resultados das avaliações indicaram que o desempenho em todas as medidas foi melhor com o IC associado ao AASI, do que o IC isoladamente, para ambos os grupos. Os autores acrescentam ainda, que as crianças que receberem IC unilateral devem ser motivadas a usar o AASI contralateral quando exista uma audição residual útil.

Enfocando o fator resíduo auditivo pesquisado neste estudo preliminar, é notável a pouca quantidade de resíduo auditivo na orelha contralateral ao IC em pacientes do HCFMUSP. Isto se deve ao fato de que o critério para a indicação do implante coclear do HCFMUSP, baseado na portaria 1278
(Ministério da Saúde, 1999) ${ }^{17}$, apresentava como critério de indicação para adultos, com surdez pós-lingual, a percepção de fala menor a $30 \%$ em apresentação aberta. Considerando-se o avanço tecnológico, atualmente o critério para indicação do implante coclear expandiu-se para percepção de sentenças em apresentação aberta menor ou igual a $40 \%$, com o uso do aparelho auditivo, sem leitura orofacial ${ }^{15}$.

O critério de seleção para IC está sofrendo alterações, os pacientes candidatos variam de perda neurosensorial de grau severo a profundo bilateral e profundo bilateral. Pesquisador ${ }^{20}$ cita que a avaliação detalhada das orelhas com testes além de audiometria e percepção de fala podem ser cruciais em relação à adaptação da bimodalidade. Novos protocolos são necessários no tratamento de pacientes com IC, quanto à conduta adequada na orelha não implantada ${ }^{24}$.

Este fato poderá elevar o número de pacientes candidatos ao uso da bimodalidade devido maior quantidade de resíduo auditivo, sendo assim, mais pacientes estarão aptos ao uso de IC associado ao AASI. Cabe aos fonoaudiólogos e otorrinolaringologistas orientarem os pacientes quanto ao uso.

\section{CONCLUSÃO}

Os pacientes do Grupo de Implante Coclear da Divisão de Otorrinolaringologia da Faculdade de Medicina da Universidade de São Paulo, que não usam AASI contralateral ao IC, têm perfil residual audiológico médio de $122.5 \mathrm{~dB}$.

Os pacientes pesquisados e que se beneficiam do uso de AASI associado ao IC tem perfil residual audiológico médio de 101,25 dB.

\section{ABSTRACT \\ Purpose: to know the audiologic profile of users and non-users of $\mathrm{HA}$ contralateral to the $\mathrm{Cl}$ in at HCFMUSP Methods: Eighteen adult, post-lingual patients were evaluated, who met the settled criteria, answering the question "Do you use or have used $\mathrm{HA}$ in a contralateral ear of a $\mathrm{Cl}$. The analysis of tonal point mean was conducted in 18 patients. Results: the patients of the Coclear Implant Group of the Division of Otolaryngology, School of Medicine of the University of Sâo Paulo, who do not use HA contralateral to the $\mathrm{Cl}$, have residual profile in average of $122.5 \mathrm{~dB}$. The patients who benefit from the use of $\mathrm{HA}$ associated with the $\mathrm{Cl}$ have residual profile in an average of $101.25 \mathrm{~dB}$. Conclusion: non- users of contralateral HA have residual hearing clinic, significantly worse than the patients who use contralateral $\mathrm{HA}$ associated with $\mathrm{Cl}$ in the Division of Otolaryngology, HCFMUSP.}

KEYWORDS: Cochlear Implantation; Hearing Aids; Hearing Loss 


\section{REFERÊNCIAS}

1. Albernaz PLM. Implante coclear. Parte 2. Rev Bras Med Otorrinolaringol. 1996; 3(2):119-22.

2. Bilger RC. Evaluation of subjects presently fitted with implanted auditory prosthesis. Ann Otol Rhinol Laryngol. 1977; 86(Suppl38):3-175.

3. Albernaz PLM, Fukuda Y, Ganaça MM, Ocougne ET, Chiarella ET, Chun CC, et al. A cirurgia na surdez total. Rev Bras Med. 1981; 38(4):164-73.

4. Bento RF, Brito Neto RV, Sanchez TG. Complicações da cirurgia do implante coclear. Arq Int Otorrinolaringol. 2001; 5(3):130-5.

5. Kozlowski L. Implantes cocleares. Carapicuíba: Pró-Fono; 1997.

6. Bento RF, Brito Neto R, Castilho AM, Goffi-Gomez MVS, Giorgi SB, Guedes MC. Resultados auditivos com o implante coclear multicanal em pacientes submetidos a cirurgia no Hospital das Clínicas da Faculdade de Medicina da Universidade de São Paulo. Rev Bras Otorrinolaringol. 2004; 70(5): 632-7.

7. Bevilacqua MC, Moret ALM, Costa Filho OA, Nascimento LT Banhara MR. Implantes cocleares em crianças portadoras de deficiência auditiva decorrente de meningite. Rev Bras Otorrinolaringol. 2003; 69(6):760-4.

8. Bess FH, Tharpe AM, Gibler AM. Auditory performance of children with unilateral sensorineural hearing loss. Ear Hear. 1986; 7(1):20-6.

9 . Feuerstein JF. Monoaural versus binaural hearing: ease of listening, word recognition, and attentional effort. Ear Hear. 1992; 13(2):80-6.

10. Offeciers E, Morera C, Müller J, Huarte A, Shallop J, Cavallé L. International consensus on bilateral cochlear implants and bimodal stimulation. Acta Oto-laryngol. 2005; 125(9):918-9.

11. Ching TYC, Psarros $C$, Hill M, Dillon H, Incerti $P$. Should children who use cochlear implants wear hearing aids in the opposite ear? Ear Hear. 2001; 22(5):365-80.

12. Hamzavi J, Pok SM, Gstoettner W, Baumgartner WD. Speech perception with a cochlear implant used in conjunction with a hearing aid in the opposite ear. Int J Audiol. 2004; 43(2):61-5.

DOI: 10.1590/S1516-18462009005000035

RECEBIDO EM: 04/07/2008

ACEITO EM: 04/02/2009

Endereço para correspondência:

Cíntia Tizue Yamaguchi

Rua Capote Valente, $4321^{\circ}$ andar

São Paulo - SP

CEP: 05409-001

E-mail: cintiay@usp.br
13. Seeber BU, Baumann U, Fastl H. Localization ability with bimodal hearing aids and bilateral cochlear implants. J Acoustic Soc Am. 2004; 116(3):1698-709.

14. Morera C, Manrique M, Ramos A, GarciaIbanez L, Cavalle L, et al. Advantages of binaural hearing provided trough bimodal stimulation via a cochlear implant and a conventional hearing aid: a 6-month comparative study. Acta Oto-Laryngol. 2005; 125(6):596-606.

15. Goffi-Gómez MVS, Guedes MC, Sant'Anna SBG, Peralta CGO, Tsuji RK, Castilho AM, et al. Critérios de seleção e avaliação médica e audiológica dos candidatos ao implante coclear: protocolo HC-FMUSP. Arq ORL. 2004; 8(4):295-314.

16. Davis $\mathrm{H}$, Silverman SR. Auditory test hearing aids. In: Davis $\mathrm{H}$, Silverman SR. Hearing and deafness. Holt: Rinehart and Winston; 1970.

17. Brasil. Ministério da Saúde, 1999. Portaria 1278. Disponível em http://portal.saude.gov.br/portal/sas/ sapd/visualizar_texto.cfm?idtxt=22689

18. Bevilacqua MC, Costa Filho OA, Nascimento LT, Banhara MR. Uso combinado do implante coclear e aparelho de amplificação sonora individual em adultos. Dist Comun. 2004; 16(1):27-33.

19. Ching TY, Hill M, Brew J, Incerti P, Priolo S, Rushbrook E, et al. The effect of auditory experience on speech perception, localization, and functional performance of children who use a cochlear implant and a hearing aid in opposite ears. Int $\mathrm{J}$ Audiol. 2005; 44(12):677-90.

20. Luntz M, Yehudai N, Shpak T. Natural history of contralateral residual hearing in binauralbimodal hearing. Acta Oto-Laryngologica. 2008; 128(12):1322-8.

21. Firszt JB, Reeder RM, Skinner MW. Restoring hearing symmetry with two cochlear implants or one implant and a contralateral hearing aid. J Rehabil Res Develop. 2008; 45(5):749-68.

22. Yuen KCP, Cao K, Wei C, Luan L, Li H, Zhang $Z$. Lexical tone and word recognition in noise of Mandarin-speaking children who use cochlear implants and hearing aids in opposite ears. Cochlear Implants Int. 2009; 10(Suppl1):120-9.

23. Ching TYC, Massie R, Van Wanrooy E, Rushbrooke E, Psarros C. Bimodal fitting or bilateral implantation? Cochlear Implants Int. 2008; 10(Suppl1):23-7.

24. Tange RA, Grolman W, Dreschler WA. What to do with the other ear after cochlear implantation. Cochlear Implants Int. 2009; 10(1):19-24. 